\title{
Summary of the National Advisory Committee on Immunization's Update on the recommended human papillomavirus (HPV) vaccine immunization schedule
}

\author{
Ismail $\mathbf{S}^{1}$, Deeks $\mathbf{S}^{2,3}$, on behalf of the National Advisory Committee on Immunization* \\ ${ }^{1}$ Public Health Agency of Canada, Centre for Immunization and Respiratory Infectious Diseases, Ottawa, ON \\ ${ }^{2}$ NACI HPV Working Group Chair, Toronto, ON \\ ${ }^{3}$ Immunization and Vaccine Preventable Diseases, Public Health Ontario, Toronto, ON \\ *Correspondence: naci-ccni@phac-aspc.gc.ca
}

\begin{abstract}
Background: Human papillomavirus (HPV) infections are the most common sexually transmitted infections. In the absence of vaccination, it is estimated that $75 \%$ of sexually active Canadians will have a sexually transmitted HPV infection at some point in their lives. Canada's National Advisory Committee on Immunization (NACl) has recommended a three-dose immunization schedule with HPV vaccine for females 9 years of age and older and for males between 9 and 26 years of age, since 2007 and 2012, respectively.
\end{abstract}

Objective: To outline the evidence on a two-dose HPV vaccine schedule and to make recommendations for the optimal HPV immunization schedule in Canada.

Methods: NACI reviewed the evidence used by the World Health Organization's (WHO's) Strategic Advisory Group of Experts (SAGE) on Immunization for the two-dose HPV immunization schedule recommended for immunocompetent girls 9 to 14 years of age and conducted an additional review of literature for studies not included in, or published after, the SAGE review. A knowledge synthesis was performed then $\mathrm{NACl}$ approved specific recommendations and elucidated the rationale and relevant considerations.

Results: Based on the evidence available to date, a two-dose HPV immunization schedule among immunocompetent 9- to 14-year-olds is expected to provide similar protective efficacy compared to a three-dose schedule in immunocompetent individuals aged 9 to 26 years. While all studies reviewed included only females, there is no reason to believe that the data would be different in males, given that data from three-dose trials demonstrates similar immune responses. Administration of two doses of HPV vaccine rather than three may increase acceptability by students, parents and health care professionals alike, and may lead to improved HPV immunization coverage and efficiencies by public health agencies. The duration of protection of either two doses or three doses of HPV vaccine is not yet known; research is encouraged to determine whether there is need for a booster dose.

Conclusion: Based on the evidence available to date, a two-dose HPV immunization schedule (given at least six months apart) among immunocompetent 9- to 14-year-olds may be considered by individuals and jurisdictions to allow for potential cost savings and other individual and programmatic advantages. A three-dose schedule should be used in individuals 15 years of age and older, as well as immunocompromised individuals and immunocompetent HIV-infected individuals. The new and complete set of current recommendations for HPV vaccines will be published in the updated HPV chapter in the Canadian Immunization Guide in the near future. 
Updated NACI recommendations on HPV vaccine (1)

\section{Recommendation \#1}

Healthy females (9 to 14 years of age)-NACI Grade A Recommendation

Either a two-dose or three-dose schedule of the HPV vaccine (Gardasil ${ }^{\circledR}$ or Cervarix ${ }^{\circledR}$ ) is recommended for immunocompetent, non-HIV infected females 9 to 14 years of age. For a two-dose schedule, at least six months between the first and second dose is recommended. If the interval between doses is shorter than five months, a third dose should be given at least six months after the first dose.

\section{Recommendation \#2}

Healthy females (15 years of age and over)—NACI Grade A Recommendation

A three-dose schedule of the HPV vaccine $\left(0,2\right.$ and 6 months for Gardasil ${ }^{\circledR}$ and 0,1 , and 6 months for Cervarix $^{\circledR}$ ) is recommended for females 15 years of age and older, unless the first dose of HPV vaccine was administered before the age of 15 years. If the first dose was administered between 9 and 14 years of age, a two-dose schedule is sufficient for females 15 years of age and older, with the second dose administered at least six months after the first dose.

\section{Recommendation \#3}

Healthy males (9 to 14 years of age)-NACI Grade B Recommendation

Either a two-dose or three-dose schedule of the HPV4 vaccine $\left(\right.$ Gardasi ${ }^{\circledR}$ ) is recommended for immunocompetent, non-HIV infected males 9 to 14 years of age. For a two-dose schedule, at least six months between the first and second dose is recommended. If the interval between doses is shorter than five months, a third dose should be given at least six months after the first dose.

\section{Recommendation \#4}

Healthy males (15 years of age and over)—NACI Grade B Recommendation

A three-dose schedule of the HPV4 vaccine (Gardasil ${ }^{\circledR} ; 0,2$ and 6 months) is recommended for males 15 years of age and older, unless the first dose of HPV vaccine was administered before the age of 15 years. If the first dose was administered between 9 and 14 years of age, a two-dose schedule is likely to be sufficient for males 15 years of age and older, with the second dose administered at least six months after the first dose.

\section{Recommendation \#5}

Immunocompromised individuals ${ }^{1}$ and immunocompetent HIV-infected individuals-NACI Grade $\mathrm{I}^{2}$ Recommendation

A three-dose schedule of the HPV vaccine (Gardasil ${ }^{\circledR}$ for males and females-0, 2, 6 months; or Cervarix ${ }^{\circledR}$ for females-0, 1, 6 months) is recommended for individuals who are immunocompromised and immunocompetent HIV-infected individuals. There is insufficient evidence to recommend a two-dose schedule in these populations; therefore, a three-dose schedule continues to be recommended for individuals who are immunocompromised and for immunocompetent HIV-infected individuals. Further study in these populations is required.

${ }^{1}$ For details on populations considered to be "immunocompromised," please refer to the chapter "Immunization in Immunocompromised Persons" in the Canadian Immunization Guide (2).

${ }^{2}$ Grade I recommendation= Insufficient evidence 


\section{References}

(1) National Advisory Committee on Immunization (NACl). Update on the recommended Human Papillomavirus (HPV) vaccine immunization schedule: An Advisory Committee Statement (ACS). 2015 Feb. http://publications.gc.ca/site/eng/477048/publication.html.

(2) National Advisory Committee on Immunization (NACl). Canadian Immunization Guide. 2015. http://www.phac-aspc.gc.ca/publicat/cig-gci/p04-hpv-vph-eng.php. 